\title{
Non-Invasive Early Detection of Oral Cancers Using Fluorescence Visualization with Optical Instruments
}

\author{
Takamichi Morikawa ${ }^{1,2, *(1)}$, Takahiko Shibahara ${ }^{1,2}$, Takeshi Nomura ${ }^{2,3}$, Akira Katakura ${ }^{2,4}$ (i) \\ and Masayuki Takano 1,2 \\ 1 Department of Oral and Maxillofacial Surgery, Tokyo Dental College, Tokyo 102-8159, Japan; \\ sibahara@tdc.ac.jp (T.S.); takano@tdc.ac.jp (M.T.) \\ 2 Oral Cancer Center, Tokyo Dental College, Chiba 272-8513; Japan; tanomura@tdc.ac.jp (T.N.); \\ katakura@tdc.ac.jp (A.K.) \\ 3 Department of Oral Oncology, Oral and Maxillofacial Surgery, Tokyo Dental College, Tokyo 102-8159, Japan \\ 4 Department of Oral Pathobiological Science and Surgery, Tokyo Dental College, Tokyo 102-8159, Japan \\ * Correspondence: morikawatakamichi@tdc.ac.jp; Tel.: +81-47-322-0151; Fax: +81-47-324-8539
}

Received: 10 August 2020; Accepted: 23 September 2020; Published: 27 September 2020

Simple Summary: Oral cancer has a high mortality rate. Then, oral cancer screening is needed for early detection and treatment. Fluorescence visualization is non-invasive, convenient, and in real-time, and examinations can be repeated. Our study aimed to show the usefulness of oral cancer screening with fluorescence visualization. A total of 502 patients were performed using fluorescence visualization that was analyzed using subjective and objective evaluation. Results of this study, subjective evaluation for detection oral cancer was high sensitivity and low specificity, while objective evaluation using imaging processing analysis was high sensitivity and high specificity. Therefore, oral cancer screening using fluorescence visualization is useful for the detection of oral cancer. The widespread use of this screening can reduce the mortality rate of oral cancer.

\begin{abstract}
Background: Oral cancer screening is important for early detection and early treatment, which help improve survival rates. Biopsy is the gold standard for a definitive diagnosis but is invasive and painful, while fluorescence visualization is non-invasive, convenient, and real-time, and examinations can be repeated using optical instruments. The purpose of this study was to clarify the usefulness of fluorescence visualization in oral cancer screening. Methods: A total of 502 patients, who were examined using fluorescence visualization with optical instruments in our hospitals between 2014 and 2019, were enrolled in this study. The final diagnosis was performed by pathological examination. Fluorescence visualization was analyzed using subjective and objective evaluations. Results: Subjective evaluations for detecting oral cancer offered $96.8 \%$ sensitivity and $48.4 \%$ specificity. Regarding the objective evaluations, sensitivity and specificity were $43.7 \%$ and $84.6 \%$ for mean green value, $55.2 \%$ and $67.0 \%$ for median green value, $82.0 \%$ and $44.2 \%$ for coefficient of variation of value, $59.6 \%$ and $45.3 \%$ for skewness, and $85.1 \%$ and $75.8 \%$ for value ratio. For the sub-analysis of oral cancer, all factors on objective and subjective evaluation showed no significant difference. Conclusions: Fluorescence visualization with subjective and objective evaluation is useful for oral cancer screening.
\end{abstract}

Keywords: oral cancer; oral squamous cell carcinoma; fluorescence visualization loss; oral potentially malignant disorders; oral cancer screening; optical Instrument; medical artificial intelligence

\section{Introduction}

Oral cancer has a high mortality rate [1,2], particularly at advanced stages, such as stage III and IV [3]. Early detection and early treatment for oral cancer are important for control [1,2]. Around $95 \%$ 
of oral cancers are oral squamous cell carcinomas (OSCCs) in histopathology [3]. In the oral cavity, ocular inspection and palpation are easily performed. Therefore, The World Health Organization (WHO) has proposed oral cancer screening mainly based on ocular inspection and palpation as a conventional oral examination (COE) to be performed by general dental practitioners (GPs) [4]. Ocular inspection is especially important for oral cancer screening. However, since various oral mucosal diseases occur in the oral cavity, it is often difficult to discriminate between them. In particular, OSCC may develop from oral potentially malignant disorders (OPMDs), such as oral lichen planus (OLP), leukoplakia, erythroplakia, and chronic candida [5]. Early detection and management of oral epithelial dysplasia (OED) in OPMDs is an important preventative step against malignant transformation, such as OSCC $[5,6]$. It is important to detect these subtle changes at an early stage.

In ocular inspection and palpation, one major limitation is the difficulty of differentiating between benign and high-risk lesions (HRL) [7], as early-stage OSCC (T1-2NOM0) cancer in situ (CIS) [5,8], and OED, may not present typical features, and a wide variety of oral mucosal diseases can present in various ways in the oral cavity [9].

Biopsy is the gold standard for definitive diagnosis. The final diagnosis is performed by pathological examination. However, this process is difficult for GPs to perform and invasive. To improve the safety and effectiveness of oral cancer screening [10], the procedure should be non-invasive or mildly invasive, simple, and repeatable. Forms of oral cancer screening include cytology [11], vital staining [12], and fluorescence visualization (FV) [13] in addition to COE.

FV has some advantages. FV is non-invasive, simple, convenient, real-time, and repeatable using optical instruments (OIs) [14]. FV uses blue light $(400-460 \mathrm{~nm})$ to illuminate flavin adenine dinucleotide (FAD), nicotinamide adenine dinucleotide (NADH), and collagen cross-links (CCL) [15-17]. The normal mucosa is visualized as apple-green autofluorescence through a selective filter with these coenzymes, and CCL. This is called fluorescence visualization retention (FVR). On the other hand, the abnormal tissues, such as HRL and inflammatory diseases, exhibit decreased autofluorescence and appear as dark brown. The appearance of this color is called FV loss (FVL) [18]. FVL is caused by the absorption of a specific wavelength of blue light due to the decreases in FAD and NADH, breakdown of CCL, and angiogenesis. However, the evaluation of FV has been visual and subjective. Therefore, definitive results have been lacking $[13,14]$.

The purpose of this study was to clarify the utility of subjective and objective evaluations using FV for oral cancer screening. Furthermore, the study aimed to improve the accuracy rate by combining them.

\section{Results}

\subsection{Patients Characteristics}

A total of 502 patients were enrolled during this study period. Of the study cohort $276(55.0 \%)$ patients were men, and $226(45.0 \%)$ were women. The tongue was the most commonly affected site ( 259 patients; $51.6 \%$ ), followed by buccal mucosa for 124 patients $(24.7 \%)$. The mean age was 62.3 years (Table S1).

Oral cancer was the most common condition, affecting 161 patients (32.1\%, including 149 patients with early OSCC, and 12 with CIS), while 235 had OPMDs (46.8\%, including 123 patients with OLP, 102 with leukoplakia, 3 with erythroplakia, and 7 with chronic candida), and 106 had other diseases (Others; 21.1\% including 32 patients with stomatitis, 24 with a benign tumor, and 50 with normal mucosa).

\subsection{Conditions}

The characteristics of each condition are showed in Table 1. The percentage of man patients with oral cancers, OPMDs, and Others was 53.4\%, 57.0\%, and 52.8\%. The mean age was $62.5,62.1$, 
and 59.9 years. The tongue was the most commonly affected site for each condition. On the control site, the subjective and objective evaluation showed no significant difference (Table 1).

Table 1. Patients' characteristics on each condition.

\begin{tabular}{|c|c|c|c|c|}
\hline & Oral Cancers & OPMDs & Others & \multirow{2}{*}{$p$-Value } \\
\hline & $n=161$ & $n=235$ & $n=106$ & \\
\hline Sex, men/women & $86 / 75$ & $134 / 101$ & $56 / 50$ & \\
\hline $\begin{array}{l}\text { Age, mean } \\
\text { Site }\end{array}$ & 62.5 & 62.1 & 59.9 & \\
\hline Tongue & 109 & 101 & 49 & \\
\hline Buccal mucosa & 20 & 89 & 15 & \\
\hline Gingiva & 22 & 37 & 14 & \\
\hline Plate & 7 & 6 & 20 & \\
\hline Others & 3 & 2 & 8 & \\
\hline \multicolumn{5}{|l|}{ Control site, mean } \\
\hline FVL rate, $(\%)$ & $0 \%$ & $0 \%$ & $0 \%$ & 1.000 \\
\hline Area, (pixels) & 927 & 967 & 962 & 0.804 \\
\hline MeanV & 81.9 & 81.7 & 80.9 & 0.224 \\
\hline MediV & 80.2 & 80.2 & 80.1 & 0.896 \\
\hline SD & 2.9 & 2.9 & 2.8 & 0.383 \\
\hline $\mathrm{CV}$ & 0.05 & 0.04 & 0.04 & 0.700 \\
\hline Skewness & 16.8 & 78.1 & 55.9 & 0.780 \\
\hline Kurtosis & -599 & -621 & -689 & 0.829 \\
\hline \multicolumn{5}{|l|}{ Lesion site, mean } \\
\hline FVL rate, $(\%)$ & $96.9 \%$ & $61.7 \%$ & $32.1 \%$ & $1.6 \times 10^{-10}$ \\
\hline Area, (pixels) & 215,408 & 160,045 & 129,048 & $9.9 \times 10^{-9}$ \\
\hline MeanV & 60.9 & 72.1 & 68.7 & 0.005 \\
\hline MediV & 58.4 & 69.8 & 68.0 & 0.007 \\
\hline $\mathrm{SD}$ & 12.6 & 9.5 & 10.1 & $9.0 \times 10^{-9}$ \\
\hline $\mathrm{CV}$ & 0.21 & 0.14 & 0.15 & $5.2 \times 10^{-12}$ \\
\hline Skewness & 0.87 & 0.28 & 0.36 & 0.107 \\
\hline Kurtosis & 2.6 & 3.3 & 6.3 & 0.082 \\
\hline VRatio, (\%) & $68.9 \%$ & $93.8 \%$ & $95.8 \%$ & $2.6 \times 10^{-17}$ \\
\hline
\end{tabular}

MeanV: mean G value, MediV: median G value, SD: standard deviation, CV: coefficient of variation, VRatio: value ratio, OPMDs: oral potentially malignant disorders.

\subsubsection{Oral Cancers}

In the histopathological diagnosis results, early OSCC grades 1, 2, and 3 were seen in $98(65.8 \%)$, $36(24.2 \%)$, and 15 patients (10.0\%). Stage I OSCC (T1N0M0) was observed in $77(51.7 \%)$ and stage II (T2N0M0) in 72 patients (48.3\%) according to the TNM Classification of Malignant Tumours 8th Edition by the Union for International Cancer Control (UICC). Visual classification [19] of oral cancers, superficial spread, exophytic, and the endophytic type was possible for $39(24.2 \%), 68(42.2 \%)$, and 54 patients (33.6\%).

A typical case of OSCC is shown in Figure 1. OSCC shows non-uniform FVL and unclear FVL borders.

In the subjective evaluation, FVR was seen in only 5 patients (3.2\%), while FVL was observed in 156 patients $(96.8 \%)$. In the objective evaluation, the area was 215,408 pixels, mean $\mathrm{G}$ value (MeanV) was 60.9 , median $G$ value (MediV) was 58.4, the standard deviation of $G$ value (SD) was 12.6, coefficient of variation of $G$ value $(C V)$ was 0.21 , skewness was 0.87 , kurtosis was 2.6 , value ratio (VRatio) was $68.9 \%$.

\subsubsection{OPMDs}

Following histopathological diagnosis, for OLP, chronic inflammation was observed 121 (98.4\%) patients and OED in 2 (1.6\%; 1 low grade and 1 high grade). Regarding leukoplakia, hyperkeratosis, OED, and papillary hyperplasia were seen in 61 (59.8\%), 31 (30.4\%; 16 low grade and 15 high grade), 
and 10 patients $(9.8 \%)$, respectively. As for erythroplakia, high-grade OED was seen in all patients. Chronic candida, Candida albicans was also detected in all patients.

Typical cases of OLP and leukoplakia are shown in Figures 2 and 3. OLP shows FV acceleration (FVA) in lace-like leukoderma and clear margin uniform FVL in erythema. Leukoplakia shows clear margin FVA in leukoderma. Most cases exhibited FVA and no FVL. Unclear FVL boundaries were observed for OED.

In the subjective evaluation, the FVL rate was $61.7 \%$. For the objective evaluation, the area was 160,045 pixels, MeanV was 72.1 , MediV was 69.8 , SD was 9.5, CV was 0.14 , skewness was 0.28 , kurtosis was 3.3 , and VRatio was $93.8 \%$.

\subsubsection{Others}

Following histopathological diagnosis, catarrhal stomatitis and viral stomatitis were seen in $22(68.7 \%)$ and 10 patients $(31.3 \%)$ with stomatitis. Patients with stomatitis did not include those with symptomatic disease, such as pemphigus, pemphigoid, or Behçet's disease. A benign tumor, fibroma and papilloma, lipoma, granular cell tumor, and solitary fibrous tumor were seen in $15(62.5 \%)$, $5(20.8 \%), 2(8.3 \%), 1(4.2 \%)$, and $1(4.2 \%)$ of the patients.

Typical cases of stomatitis and benign tumor are shown in Figures 4 and 5 . Stomatitis shows uniform FVL around the central ulcer. In the surface plot, a clear boundary in the center and uniform FVA and a uniform FVL in the periphery are observed. The benign tumor showed FVR alone and no FVL.

In the subjective evaluation, FVL rate was $32.1 \%$. In the objective evaluation, the area was 129,048 pixels, MeanV was 68.7, MediV was 68.0 , SD was $10.1, \mathrm{CV}$ was 0.15 , skewness was 0.36 , kurtosis was 6.3, and VRatio was $95.8 \%$.

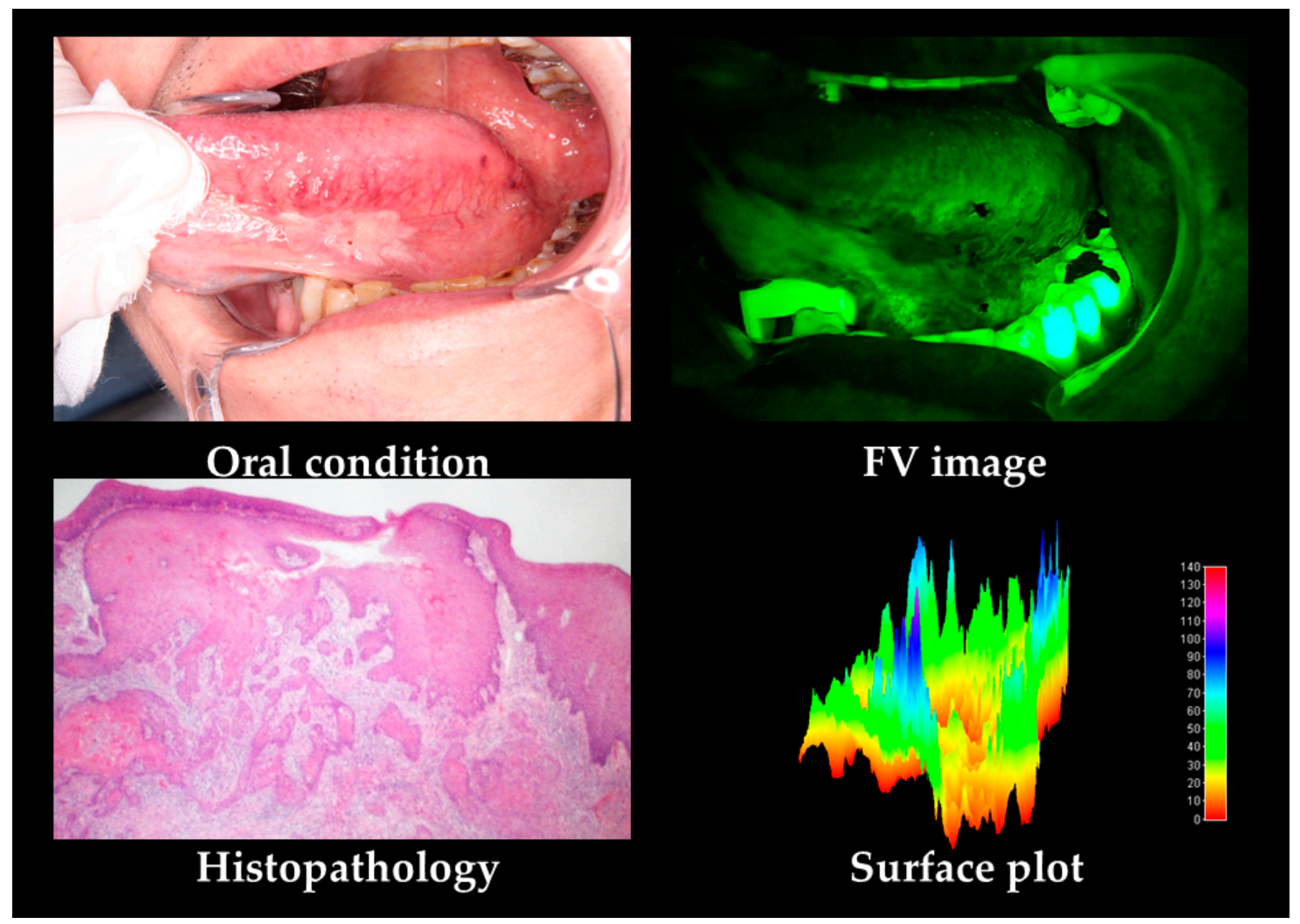

Figure 1. Grade 1 oral squamous cell carcinomas (OSCC) case according to histopathological features. As OSCC shows non-uniform and unclear margin fluorescence visualization loss (FVL). In the surface plot, non-uniform and unclear borders of decreased value are observed. 


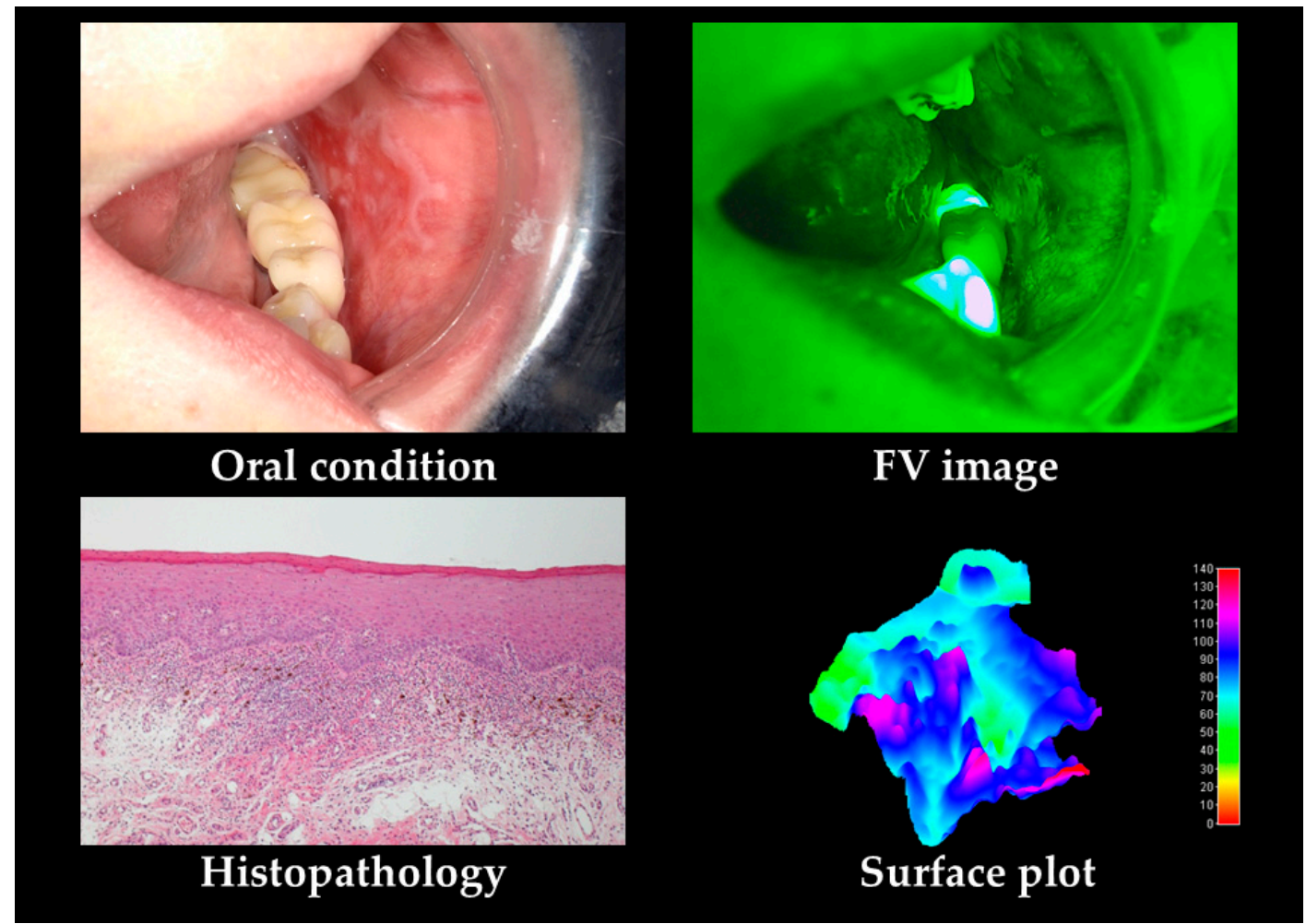

Figure 2. Oral lichen planus (OLP) case. Histopathological features include epithelial parakeratosis and zonal infiltration of subepithelial inflammatory cells. OLP shows fluorescent visualization acceleration (FVA) in lace-like leukoderma and clear margin, uniform FVL in erythema. In the surface plot, part of the erythema shows a decrease, and part of the lace-like leukoderma shows an increase.

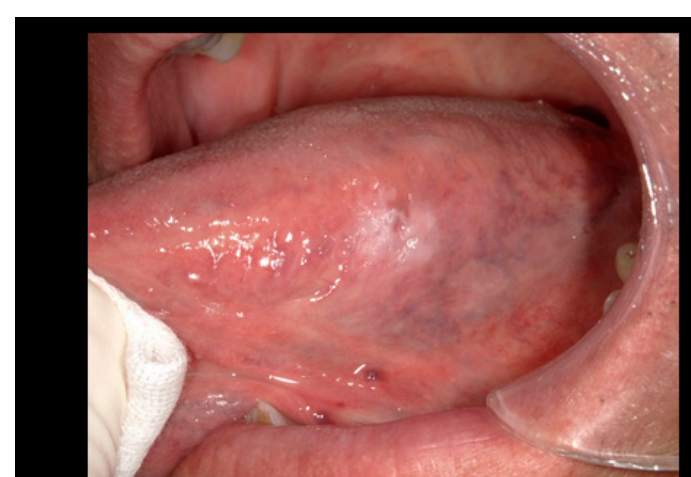

Oral condition

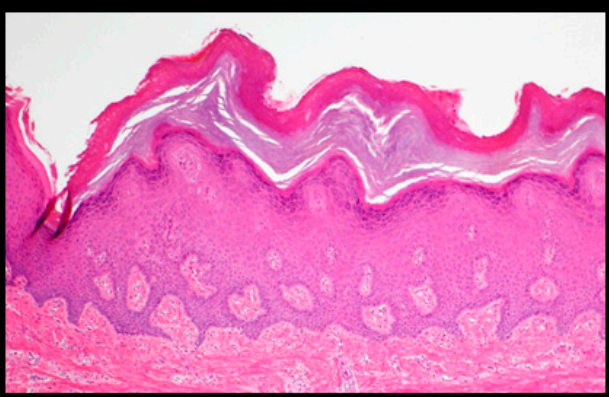

Histopathology

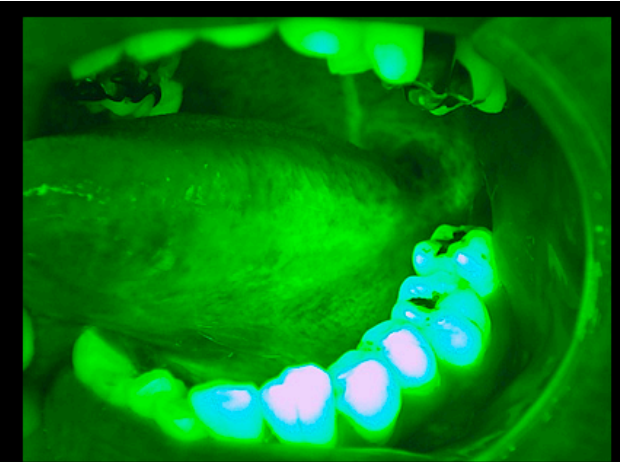

FV image

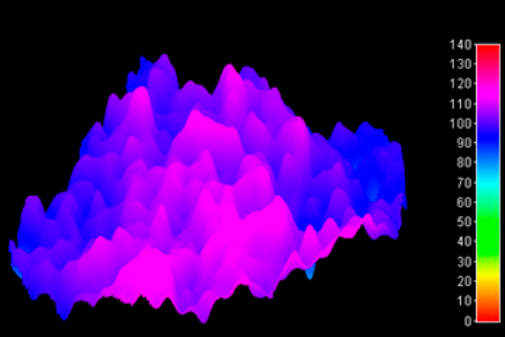

Surface plot

Figure 3. Leukoplakia case. The main histopathological feature is hyperplasia. The figure shows FVA alone and no FVL. In the surface plot, a clear boundary and uneven acceleration are observed. 


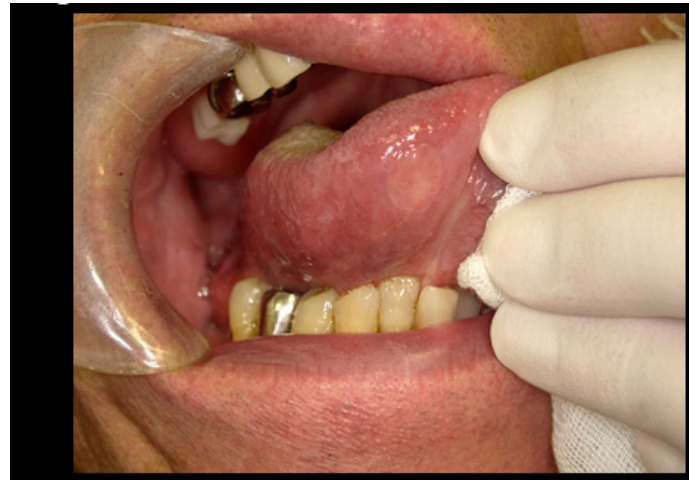

Oral condition

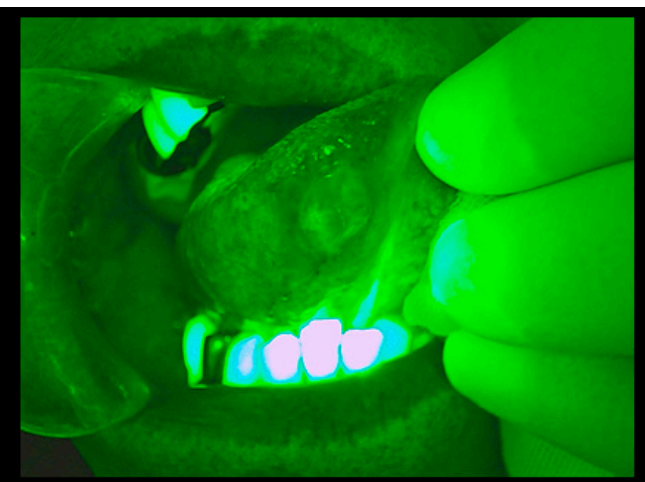

FV image

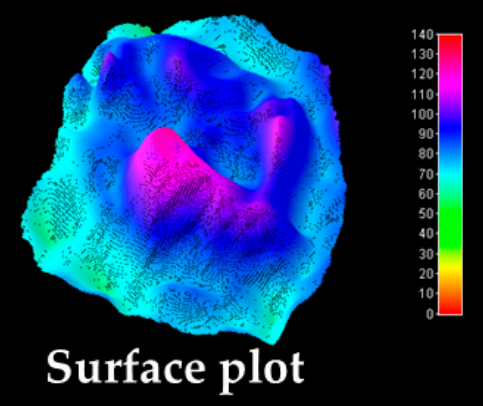

Figure 4. Stomatitis case. Stomatitis shows concentric narrow, clear margin, and uniform FVL around the central ulcer. In the surface plot, a clear boundary in the center and uniform FVA and a uniform FVL in the periphery are observed.
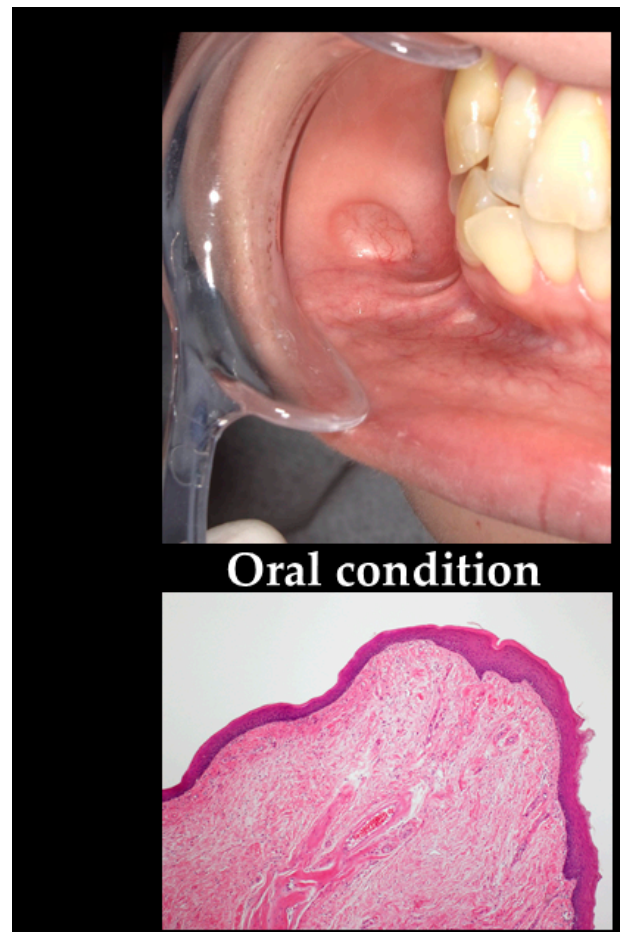

Histopathology

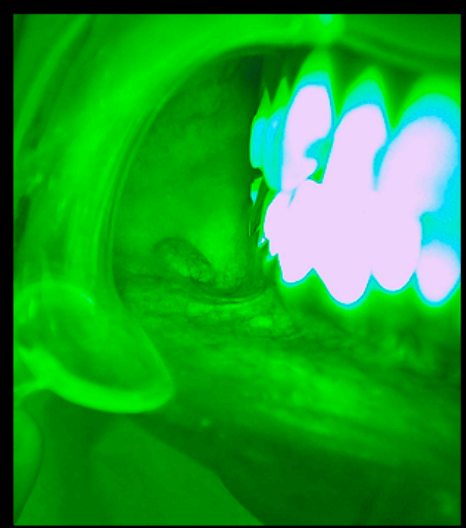

FV image

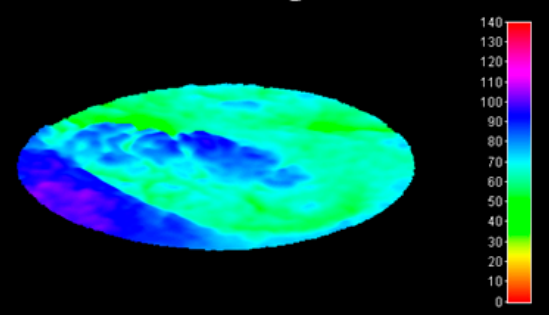

Surface plot

Figure 5. Fibroma as a benign tumor. Fibroma shows FVR alone and no FVL. The surface plot was observed to be flat. 


\subsection{Analysis}

\subsubsection{Comparison between Each Group}

In the subjective evaluation, the highest rate was found for oral cancers $\left(p=1.6 \times 10^{-10}\right)$.

Results of objective evaluation showed that oral cancers were the conditions largest in area $\left(p=9.9 \times 10^{-9}\right)$; lowest in MeanV and MediV ( $p=0.005$ and 0.007); and highest in SD and CV $\left(p=9.0 \times 10^{-9}\right.$ and $\left.5.2 \times 10^{-12}\right)$. Skewness and kurtosis were not significant $(p=0.107$ and 0.082$)$. Oral cancers had the lowest VRatio $\left(p=2.6 \times 10^{-17}\right)$.

\subsubsection{Detection of Oral Cancers}

In subjective evaluation, sensitivity and specificity were $96.8 \%$ and $48.4 \%\left(p=1.4 \times 10^{-23}\right)$.

Table 2 shows the objective evaluation for detection of oral cancers by receiver operating characteristic (ROC) curve analysis. The area under the curve (AUC) was 0.578 in area, 0.651 in MeanV, 0.633 in MediV, 0.512 in SD, 0.820 in CV, 0.651 in skewness, 0.513 in kurtosis, and 0.827 in VRatio (Figure S1). MeanV and MediV, CV, skewness, and VRatio were significant factors, and cut-off was set at 52.5 in MeanV, 60.0 in MediV, 0.15 in CV, 0.71 in skewness, and 87.5\% in VRatio. Sensitivity and specificity were $43.7 \%$ and $84.6 \%$ in MeanV, $55.2 \%$ and $67.0 \%$ in MediV, $82.1 \%$ and $69.4 \%$ in CV, 59.6\% and $63.0 \%$ in skewness, and $85.1 \%$ and $75.8 \%$ in VRatio (Table 2).

Table 2. Object evaluation from receiver operating characteristic (ROC) analysis for detection oral cancers.

\begin{tabular}{ccccccc}
\hline & AUC & $p$-Value & Cut-off & Sensitivity & Specificity & 95\% CI \\
\hline Area & 0.578 & 0.066 & - & - & - & $0.494,0.663$ \\
\hline MeanV & 0.651 & $5.0 \times 10^{-4}$ & 52.5 & 43.7 & 84.6 & $0.570,0.732$ \\
\hline MediV & 0.633 & 0.002 & 60.0 & 55.2 & 67.0 & $0.551,0.715$ \\
\hline SD & 0.512 & 0.779 & - & - & - & $0.427,0.597$ \\
\hline CV & 0.820 & $8.4 \times 10^{-5}$ & 0.15 & 82.1 & 69.4 & $0.782,0.858$ \\
\hline Skewness & 0.651 & $4.2 \times 10^{-4}$ & 0.71 & 59.6 & 63.0 & $0.589,0.747$ \\
\hline Kurtosis & 0.513 & 0.707 & - & - & - & $0.570,0.731$ \\
\hline VRatio & 0.827 & $5.3 \times 10^{-14}$ & 87.5 & 85.1 & 75.8 & $0.763,0.890$ \\
\hline
\end{tabular}

ROC: Receiver operating characteristic, AUC: area under the curve, CI: confidence interval

As the result of multivariate analysis, subjective evaluation and CV, VRatio was a significant factor; Subjective evaluation, $p=0.004$, odds ratio $(\mathrm{OR})=13.866$, and 95\% confidence interval $(\mathrm{CI})=3.098$, 62.084; $\mathrm{CV}, p=0.005, \mathrm{OR}=5.454,95 \% \mathrm{CI}=2.871,10.360$; VRatio, $p=0.001, \mathrm{OR}=5.642,95 \% \mathrm{CI}=2.928$, 10.871 (Table 3).

Table 3. Multivariate analysis for detection oral cancers.

\begin{tabular}{ccccc}
\hline & Univariate & \multicolumn{3}{c}{ Multivariate } \\
\hline & $p$-Value & $p$-Value & OR & 95\% CI \\
\hline Subjective evaluation & $1.4 \times 10^{-23}$ & 0.004 & 13.866 & $3.098,62.084$ \\
MeanV & $5.0 \times 10^{-4}$ & 0.329 & 1.066 & $0.814,1.071$ \\
MediV & 0.002 & 0.321 & 1.073 & $0.933,1.233$ \\
CV & $8.4 \times 10^{-5}$ & 0.005 & 5.454 & $2.871,10.360$ \\
Skewness & $4.2 \times 10^{-4}$ & 0.357 & 1.238 & $0.788,1.938$ \\
VRatio & $5.3 \times 10^{-14}$ & 0.001 & 5.642 & $2.928,10.871$ \\
\hline
\end{tabular}

OR: odds ratio. 


\subsubsection{Combination of Each Factor for Detection of Oral Cancer}

Each item was combined for subjective evaluation, and CV and VRatio were evaluated. Each OR was scored by the following calculation formula.

Combination for detection of oral cancer $=13.9 \times$ Subjective evaluation $+5.5 \times \mathrm{CV}+5.6 \times$ VRatio (minimum score 0, maximum score 25)

Using ROC curve analysis, the combination for the detection of oral cancer was set; the AUC was 0.891 , and the cut-off was set at 19.4. Sensitivity and specificity were $86.6 \%$ and $84.6 \%$ (Figure S2).

\subsubsection{Detection of OPMDs}

In the subjective evaluation, sensitivity and specificity were $58.5 \%$ and $26.4 \%$ for detection of OPMDs $\left(p=3.3 \times 10^{-4}\right)$.

The objective evaluation for detection of OPMDs by ROC curve analysis is shown Table 4 . The AUC was 0.450 in area, 0.602 in MeanV, 0.601 in MediV, 0.632 in SD, 0.740 in CV, 0.632 in skewness, 0.523 in kurtosis, and 0.767 in VRatio (Figure S3). MeanV and MediV, SD, CV, skewness, and VRatio were significant factors. Cut-off was set at 80.0 in MeanV, 50.0 in MediV, 12.9 in SD, 0.17 in CV, 0.56 in skewness, and $74.5 \%$ in VRatio. Sensitivity and specificity were $39.9 \%$ and $74.8 \%$ in MeanV, $81.3 \%$ and $36.8 \%$ in MediV, $83.5 \%$ and $38.1 \%$ in SD, $80.2 \%$ and $56.7 \%$ in CV, $68.8 \%$ and $64.8 \%$ in skewness, and $82.7 \%$ and $55.7 \%$ in VRatio.

Table 4. Objective evaluation from ROC analysis for the detection oral potentially malignant disorders (OPMDs).

\begin{tabular}{ccccccc}
\hline & AUC & $p$-Value & Cut-off & Sensitivity & Specificity & 95\% CI \\
\hline Area & 0.450 & 0.063 & - & - & - & $0.397,0.502$ \\
\hline MeanV & 0.602 & $1.7 \times 10^{-5}$ & 80.0 & 39.9 & 74.8 & $0.551,0.653$ \\
\hline MediV & 0.601 & 0.020 & 50.0 & 81.3 & 36.8 & $0.518,0.684$ \\
\hline SD & 0.632 & $1.0 \times 10^{-6}$ & 12.9 & 83.5 & 38.1 & $0.580,0.683$ \\
\hline CV & 0.740 & $7.5 \times 10^{-19}$ & 0.17 & 80.2 & 56.7 & $0.695,0.786$ \\
\hline Skewness & 0.632 & 0.002 & 0.56 & 68.8 & 64.8 & $0.551,0.713$ \\
\hline Kurtosis & 0.523 & 0.585 & - & - & - & $0.440,0.607$ \\
\hline VRatio & 0.767 & $1.4 \times 10^{-10}$ & 74.5 & 82.7 & 55.7 & $0.619,0.712$ \\
\hline
\end{tabular}

As seen the results of multivariate analysis, subjective evaluation, $\mathrm{CV}$, and VRatio were significant factors (subjective evaluation, $p=1.6 \times 10^{-4}, \mathrm{OR}=0.089$, and 95\% $\mathrm{CI}=0.025,0.314 ; \mathrm{CV}, p=0.012$, $\mathrm{OR}=0.001,95 \% \mathrm{CI}=0.000001,0.188 ;$ VRatio, $p=0.031, \mathrm{OR}=1.024,95 \% \mathrm{CI}=1.002,1.046$; Table 5).

Table 5. Multivariate analysis for detection OPMDs.

\begin{tabular}{ccccc}
\hline & Univariate & \multicolumn{3}{c}{ Multivariate } \\
\hline & $p$-Value & $p$-Value & OR & $\mathbf{9 5 \% ~ C I ~}$ \\
\hline Subjective evaluation & $3.3 \times 10^{-4}$ & $1.6 \times 10^{-4}$ & 0.089 & $0.025,0.314$ \\
MeanV & $1.7 \times 10^{-4}$ & 0.249 & 1.078 & $0.949,1.225$ \\
MediV & 0.020 & 0.214 & 0.926 & $0.821,1.045$ \\
SD & $1.0 \times 10^{-6}$ & 0.933 & 1.012 & $0.764,1.341$ \\
CV & $7.5 \times 10^{-19}$ & 0.012 & 0.001 & $0.000001,0.188$ \\
Skewness & $0.002^{4}$ & 0.700 & 0.933 & $0.656,1.327$ \\
VRatio & $1.4 \times 10^{-10}$ & 0.031 & 1.024 & $1.002,1.046$ \\
\hline
\end{tabular}

\section{Discussion}

Early diagnosis by GPs is considered likely to improve the outcomes of oral cancer. A biopsy is the gold standard method of definitive diagnosis for oral cancer. However, the procedure is highly 
invasive, and painful. GPs may require specialized training to perform a biopsy, and the dissemination of cancer cells into the circulation results in an increased risk of metastasis after the biopsy [20,21]. Screening should be minimally invasive, inexpensive, and repeatable. Cytology [11], vital staining [12], and FV [13-19] are simple and effective methods of screening for oral cancer.

Oral liquid-based brush cytology (OLBC) is an approach that involves collecting cells from the oral mucosa using a brush technique and creating homogeneous slides, and this method in efficient in cell collection [22]. OLBC is easy to use, relatively painless, and well-accepted by patients [23]. It was reported that $69.0 \%$ and $29.0 \%$ of patients feel discomfort and pain, respectively, with OLBC [24]. Several days are required to obtain results from OLBC. OLBC is also not a reliable means of evaluating lesions with thick keratin layers [22]. According to reports [11,22-26], the sensitivity and specificity are $75.0 \%$ and $50.0 \%$ [22], and $86.5 \%$ and $94.3 \%$ [26].

Vital staining is a method that can detect suspicious lesions using iodine solution (IS) and toluidine blue (TB) [12]. Vital staining with IS and TB in the oral cavity allows easy observation of the results in real-time. In OSCC, CIS, and low- and high-grade OED, little glycogen is present in the granule cell layer, resulting in iodine unstained (IU) areas [12,27]. However, IS cannot be used for patients with iodine allergy, and the technique is invasive [28]. IS is applicable for movable mucosae such as the tongue, oral floor, buccal mucosa, and soft palate, but is difficult to use on keratinized mucosa such as the gingiva and hard palate [10]. It was reported that $53.1 \%$ and $21.4 \%$ of patients feel discomfort and pain, respectively, with IS [28]. According to reports [12,27-29], the sensitivity and specificity for detection oral cancer are $56.0 \%$ and $46.8 \%$ [29], and 88.5 and 51.6\% [12]. TB binds to the acidic groups of acidic mucopolysaccharides, such as nucleic acids of DNA and RNA, and exhibits metachromasia, dark royal blue relative to OSCC and CIS, a pale blue on high-grade OED, and unstained on normal mucosa, depending on nucleic acid content [12,30]. However, TB has invasive aspects such as sourness and bitterness and an acetic acid odor. TB is hazardous if swallowed and was shown to have toxicity to fibroblasts [31]. Inflammatory lesions bind TB, and this can contribute to false-positive outcomes. TB is applicable for all oral mucosa in detecting only oral cancer, but it is debatable whether OED can be detected with this method [32]. TB is also not reliable for evaluating lesions with thick keratin layers [30]. According to reports $[12,33,34]$, the sensitivity and specificity for detection oral cancer are $80.8 \%$ and $96.7 \%$ [12], and $100 \%$ and $36,9 \%$ [35].

FV with OIs is simple and non-invasive, convenient, and examinations can be repeated $[13,14]$. The results of FV are available in real-time. It can be adapted to any area of the oral cavity (Figure 6). There are various types of OIs, such as VELscope ${ }^{\circledR}$ (LED Medical Diagnostics, White Rock, BC, Canada) [35], ViziLite ${ }^{\circledR}$ (Zila Pharmaceuticals, Phoenix, AZ, United States), ORALOOK ${ }^{\circledR}$ (HITS PLAN, Tokyo, Japan), and IllumiScan ${ }^{\circledR}$ (SHOFU, Kyoto, Japan) [13]. ORALOOK ${ }^{\circledR}$ is a lightweight and simple instrument; it has a built-in filter, while FV images and oral conditions are captured using one unit [7]. This OI irradiates the target with a blue light at an excitation wavelength of about $422 \mathrm{~nm}$, while it can detect only apple-green fluorescence via a filter $(520-560 \mathrm{~nm})$. IllumiScan ${ }^{\circledR}$ is a lightweight and simple instrument; it has a built-in filter, and only FV images are captured using one unit [16]. This OI's excitation wavelength is $425 \mathrm{~nm}$, its filter is $470-580 \mathrm{~nm}$, and it can detect only apple-green fluorescence. These are the OIs that we developed jointly with our departments.

FV can enhance the visibility of oral mucosal abnormalities by activating tissue autofluorescence. Autofluorescence is due to the presence of endogenous fluorophores in cells, such as FAD, NAD, and CCL, which produce a fluorescent emission when exposed to the light of a specific wavelength [36]. The denaturation and destruction of CCL by dysplastic progression cause FVL [37]. The fluorescence intensity due to FAD decreases with the progression of dysplasia [14]. FAD and NADH are important fluorophores that become excited at those wavelength intervals in epithelium cells. These coenzymes are known to involve different types of intracellular energy metabolism, such as glycolysis, the tricarboxylic acid cycle, and the electron transport chain [38,39]. It is known that dysplastic progression enhances a form of anaerobic metabolism called the Warburg effect [40]. Since FAD and NADH are intermediate enzymes, they are consumed when anaerobic metabolism is enhanced, and, as a result, 
the autofluorescence decreases. FVL occurs because the wavelength of blue light is absorbed by hemoglobin. Furthermore, angiogenesis occurs due to cell proliferation [41], and an inflammatory response is triggered by the immune response, resulting in FVL [42].

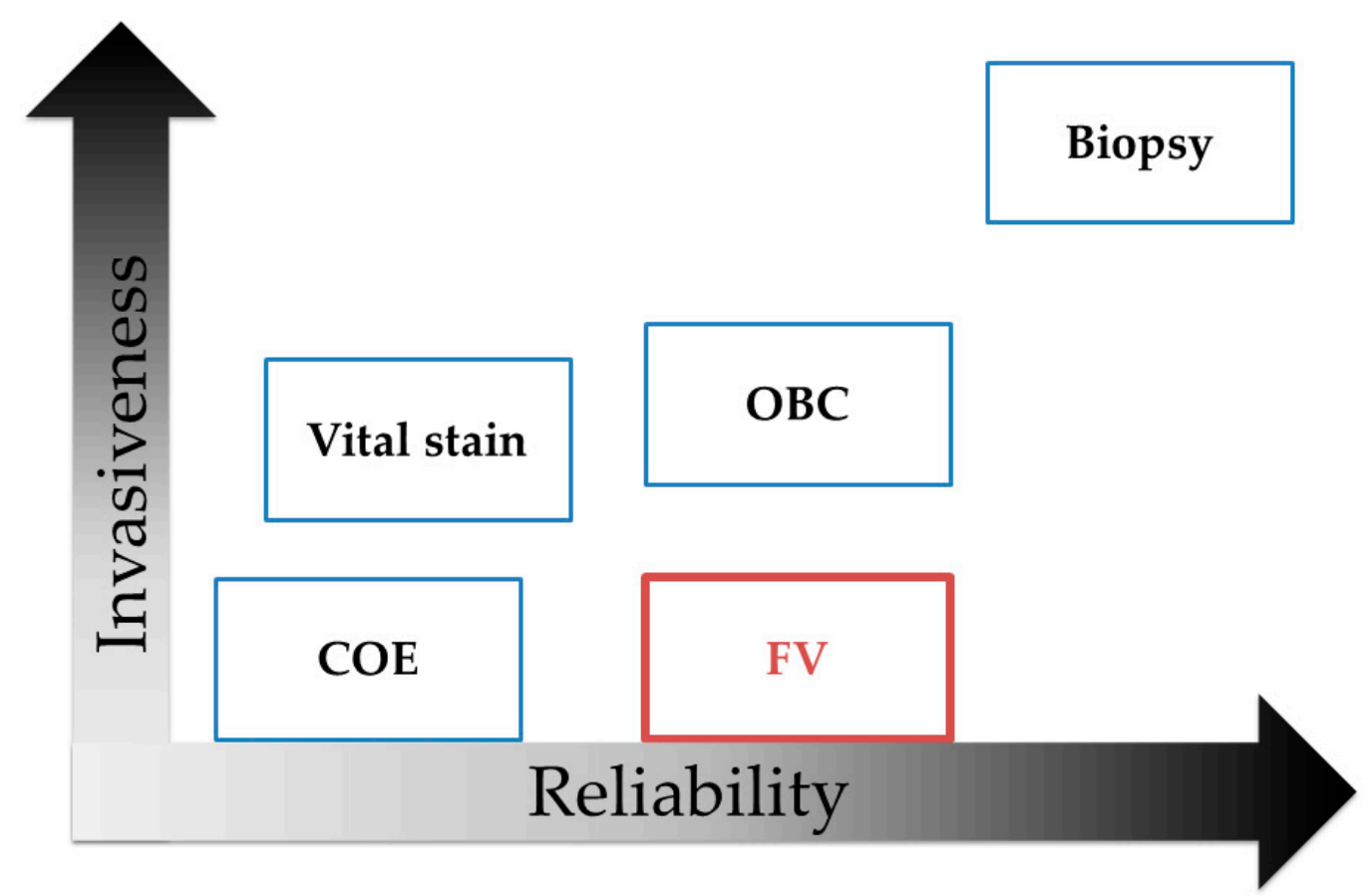

Figure 6. The concept of oral cancer screening in terms of invasiveness and reliability. Biopsy is the gold standard method for oral cancer screening. Conventional oral examination (COE) and FV are non-invasive methods, and FV is of high reliability.

Various accuracy levels have been reported; the sensitivity and specificity for detection oral cancer were $33.3 \%$ and $88.6 \%$ [42], $100 \%$ and $80.8 \%$ [43], and $91.0 \%$ and $100 \%$ [44]. The result of subjective evaluation in this study showed high sensitivity $(96.8 \%)$ and low specificity $(48.4 \%)$. As this difference in accuracy was for subjective evaluation, it was considered that there is a large difference between evaluators. Therefore, in this study, we performed subjective and objective evaluation by using image processing analysis.

RGB is a color representation method. Various colors can be represented by the three primary colors of Red, Green, and Blue [45]. When the dominant wavelength of the light is in the upper end of the visible spectrum, it is perceived as red, and if it is in the lower end, it is defined as blue [46]. The human eye is more sensitive to green because the ratio of luminosity function is $555 \mathrm{~nm}$ for humans [47]. Hence, the OIs used in this study were selected because they use a single green fluorescence color ( $\mathrm{G}$ value), and image processing analysis is simple.

As a result of objective evaluation in this study, we set various factors, such as MeanV and MediV. MeanV was reported to contribute to the detection of oral cancer $[15,16,48]$. On the other hand, Kozakai also reported that it is difficult to distinguish oral cancer from OLP [49]. In this study, MeanV (60.9) and MediV (58.4) for oral cancers were lower than for OPMDs (MeanV 72.1, MediV 69.8) and Others (MeanV 68.7, MediV 68.0). The AUC of MeanV and MediV was 0.651 and 0.633 . The sensitivity and specificity were $43.7 \%$ and $84.7 \%$ for MeanV, $55.2 \%$ and $67.0 \%$ for MediV. The results showed high specificity and low sensitivity.

The variation was evaluated by SD and CV. SD is difficult to compare if the means are different. On other hand, $\mathrm{CV}$ can relatively evaluate the relationship of variation. Oral cancer is a highly variable disease due to the heterogeneity within the tumor [50-52]. Kosugi et al. reported that the G value had more variation and a wider range with increasing tumor progression using an animal tongue 
carcinogenesis model [52]. In addition, they considered that tumor progression can be monitored by this new $G$ value analysis method in humans. In this study, CV in oral cancers (0.21) was high compared with other diseases (OPMDs 0.14, Others 0.15). The AUC of CV was 0.820. The sensitivity and specificity were $82.1 \%$ and $69.4 \%$ for CV. The results showed high sensitivity and high specificity.

The skewness and kurtosis show the distribution. There have been no reports on skewness and kurtosis in FV image processing analysis. In oral cancers, the skewness and kurtosis varied greatly depending on the case. Skewness for oral cancers was high compared with that for OPMDs and Others. Kurtosis for oral cancers was low compared with that for OPMDs and Others. The AUC of skewness and kurtosis were 0.651 and 0.513 . The sensitivity and specificity were $59.6 \%$ and $45.3 \%$ for skewness.

FV is greatly affected by environmental factors such as natural light $[45,53]$. In this study, we tried to maintain the same conditions as much as possible. Therefore, the cases were in a state where there was no significant difference (Table 1, control). However, it is difficult to eliminate environmental factors completely in clinical practice. Therefore, in this study, we corrected it by using a normal mucosa as a control, represented by VRatio [7,15-17,49]. In this study, VRatio for oral cancers $(68.9 \%)$ lower than that of OPMDs and Others (OPMDs 93.8\%, Others 95.8\%). The AUC of the VRatio was 0.827. The sensitivity and specificity were $85.1 \%$ and $75.8 \%$ on VRatio. There were high sensitivity and high specificity. The results of multivariate analysis for the detection of oral cancer showed that subjective evaluation, CV, and VRatio were significant factors. These combinations were effective for the detection of oral cancer.

In this study, OIs were used randomly. These OIs can be used equally in both subjective and objective evaluations [54]. It was considered that this semi-quantification would enable more accurate diagnosis assistance without being influenced by the evaluator. This study may be able to aid remote diagnostics, especially in under-developed areas and developing countries. Many reports have described the potential application of artificial intelligence (AI) to medical fields in recent years [55]. Using this semi-quantitative evaluation, development into Medical AI is expected [54]. FV can be used in any subsite [56].

There are several limitations to our study. First, this study used a small number of patients and was retrospective. Second, a detailed examination of OED was not conducted. OED is at risk of malignant transformation and is HRL. It is also necessary to consider the detection of HRL [7]. Therefore, we are planning prospective studies for the detection of HRL and treatments for other oral subsites.

FV is a non-invasive method for early detection of oral cancer. It is effective for oral cancer screening in addition to COE, vital staining, and OBC.

\section{Materials and Methods}

A total of 502 patients were enrolled at Tokyo Dental College Chiba Hospital and Chiba Dental Center from January 2014 to December 2019. The final diagnosis was performed by pathological examination. The inclusion criteria were: (1) COE and FV were performed before treatment, (2) informed consent to participate in this study was provided, and (3) confirmed diagnosis was obtained by biopsy, except for stomatitis and normal mucosa. All patients with OSCC were re-staged using the 8th edition of the TNM staging system by the UICC $[5,8]$. The study was approved by the Ethics Review Committee of Tokyo Dental College (740) and performed in accordance with the requirements of the Declaration of Helsinki (64th WMA General Assembly, Fortaleza, Brazil, October 2013).

OIs randomly used in this study were ORALOOK ${ }^{\circledR}$ and IllumiScan ${ }^{\circledR}$. This was left to the attending physician. These OIs allowed FV images to be obtained and saved to the camera as digital data. FV images of the lesion can be observed in real-time because the OIs have their monitor. FV images were taken a room that was as dark as possible. The irradiating light was set perpendicular to the lesion and the irradiation range was set to about $10 \mathrm{~cm}$ [15-17].

In subjective evaluations, oral conditions and FV images were compared to evaluate FVR, FVA or FVL in lesions (Figure 7A,B) [57,58]. Image processing analysis was performed on the FV images using 
Image J software version 1.5 (National Institutes of Health, Bethesda, Maryland, USA) [59,60]. Regions of interest (ROIs) for the lesion and control area were established in FV images [15-17].

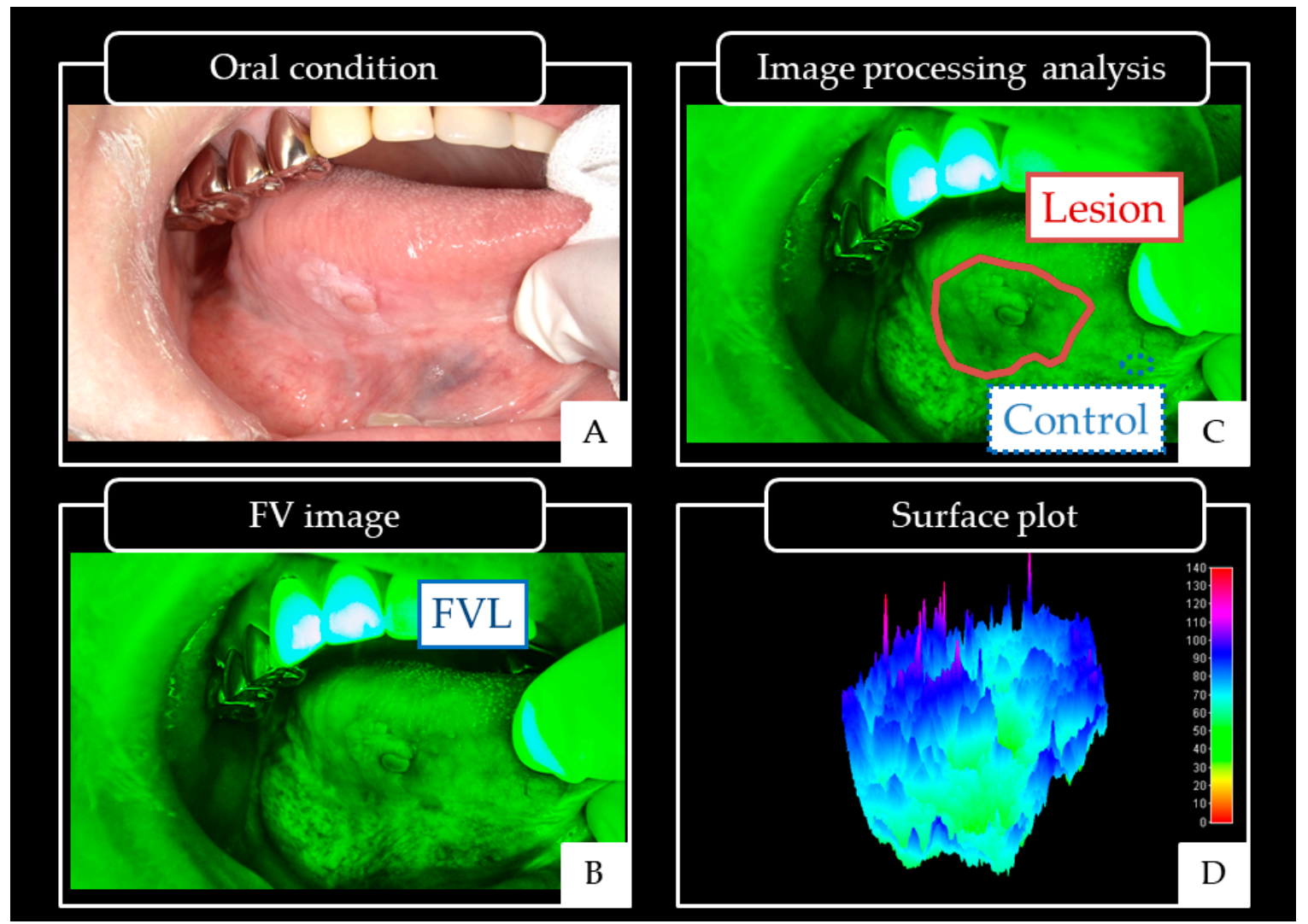

Figure 7. Oral conditions and FV image of cancer on the right side of the tongue.

The ROI of the lesion was defined as the area of FVL. The control ROI was set at a distance away in the same sub-site of the oral cavity in normal mucosa in front of the lesion without FVL and oral mucosal lesions (Figure 7C). The state of the lesion ROI was expressed in the surface plot, and color mapping was performed for the lesion ROI (Figure 7D). In subjective evaluation, we defined a positive case as FVL and a negative case as only FVA or FVR by two or more screenings using the surface plot as a reference [15-17]. The FVL rate was calculated. Sensitivity and specificity were calculated as follows; [sensitivity $=$ (FVL positive cases of oral cancers/all cases of oral cancers) $\times 100 \%$ ], [specificity $=($ FVL negative cases without oral cancers/all cases without oral cancers $) \times 100 \%]$.

For differential diagnosis of oral mucosal diseases, the chi-squared test, Mann-Whitney U test, and Fisher's exact test were used for statistical analyses. Cut-off values were set using an ROC curve to detect oral cancers and OPMDs. AUC, sensitivity, and specificity were calculated by ROC analysis. All statistical analyses were performed using SPSS version 27.0 (IBM, Tokyo, Japan). Values of $p<0.05$ were deemed statistically significant. For the multivariate analysis, the factors that were significant in the univariate analysis were used. OR and $95 \%$ CI were calculated. During the combination of each factor for the detection of oral cancers, significant factors were extracted from the results of multivariate analysis. Each factor was weighted with reference to the OR, and the effectiveness of the combination was verified.

\section{Conclusions}

FV is a non-invasive method for the early detection of oral cancers. Subjective analysis showed high sensitivity and low specificity, and objective analysis resulted in high sensitivity and high specificity for the detection of oral cancers. 
Supplementary Materials: The following are available online at http://www.mdpi.com/2072-6694/12/10/2771/s1, Figure S1: ROC for the detection of oral cancers. Figure S2: Combination of each factor for the detection of oral cancers. Figure S3: ROC for detection OPMDs. Table S1: Patients characteristics.

Author Contributions: T.M.: conceptualization, validation, resources, and data curation, formal analysis and investigation, writing-original draft preparation, project administration, and funding acquisition; T.S.: conceptualization, validation, visualization, project administration, and funding acquisition; T.N.: writing - review and editing; A.K.: writing - review and editing; M.T.: methodology, software, validation, resources, and data curation, writing-review and editing, and supervision. All authors have read and agreed to the published version of the manuscript.

Funding: This work was supported by Shofu Inc (Kyoto, Japan) and HITS PLAN Inc (Tokyo, Japan). The sponsors lent the IllumiScan ${ }^{@}$ and ORALOOK ${ }^{@}$ to our department for this study. The funding source had no involvement with regard to study design, collection, analysis, and interpretation of data, writing of the report, or regarding the decision to submit the article for publication.

Acknowledgments: The authors acknowledge the Department of Oral and Maxillofacial Surgery, the Department of Oral Pathobiological Science and Surgery, the Department of Clinical Pathophysiology, and staff support provided by Tokyo Dental College.

Conflicts of Interest: All authors declare a conflict of interest. A part of this study was funded by Shofu inc (Kyoto, Japan). T.M. and T.S. received a Lecture fee from HITS PLAN Inc (Tokyo, Japan). The funding source had no involvement with regard to study design, collection, analysis, and interpretation of data, writing of the report, or regarding the decision to submit the article for publication.

\section{Abbreviations}

\begin{tabular}{|c|c|}
\hline OSCC & Oral squamous cell carcinoma \\
\hline WHO & World Health Organization \\
\hline $\mathrm{COE}$ & Conventional oral examination \\
\hline GP & General dental practitioner \\
\hline OPMDs & Oral potentially malignant disorders \\
\hline OLP & Oral lichen planus \\
\hline OED & Oral epithelial dysplasia \\
\hline HRL & High-risk lesions \\
\hline CIS & Cancer in situ \\
\hline FV & Fluorescence visualization \\
\hline OI & Optical instrument \\
\hline FAD & Flavin adenine dinucleotide \\
\hline NADH & Nicotinamide adenine dinucleotide \\
\hline $\mathrm{CCL}$ & Collagen cross-links \\
\hline FVR & Fluorescence visualization retention \\
\hline FVL & Fluorescence visualization loss \\
\hline MeanV & Mean G value \\
\hline MediV & Median G value \\
\hline SD & Standard deviation \\
\hline $\mathrm{CV}$ & Coefficient of variation \\
\hline VRatio & Value ratio \\
\hline UICC & Union for international cancer control \\
\hline FVA & Fluorescence visualization acceleration \\
\hline $\mathrm{ROC}$ & Receiver operating characteristic \\
\hline AUC & Area under the curve \\
\hline OR & Odds ratio \\
\hline $\mathrm{CI}$ & Confidence interval \\
\hline OLBC & Oral liquid-based brush cytology \\
\hline IS & Iodine solution \\
\hline $\mathrm{TB}$ & Toluidine blue \\
\hline IU & Iodine unstained \\
\hline
\end{tabular}




\section{References}

1. Cheraghlou, S.; Schettino, A.; Zogg, C.K.; Judson, B.L. Changing prognosis of oral cancer: An analysis of survival and treatment between 1973 and 2014. Laryngoscope 2018, 128, 2762-2769. [CrossRef]

2. Japan Society for Head and Neck Cancer, Cancer Registry Committee. Report of Head and Neck Cancer Registry of Japan. Five-Year Prognosis of Patients Registered in 2011. Available online: http://www.jshnc. umin.ne.jp/pdf/2011syourei_yogo.pdf (accessed on 25 September 2020).

3. Japan Society for Head and Neck Cancer, Cancer Registry Committee. Report of Head and Neck Cancer Registry of Japan Clinical Statistics of Registered Patients. 2016. Available online: http://www.jshnc.umin.ne. jp/pdf/2016syourei_houkoku.pdf (accessed on 6 April 2020).

4. World Health Organization. Oral Cancer. Available online: https://www.who.int/cancer/prevention/ diagnosis-screening/oral-cancer/en/ (accessed on 6 April 2020).

5. El-Naggar, A.K.; Chan, J.; Takata, T.; Grandis, J.; Blootweg, P. Pathology and Genetics of Head and Neck Tumors. In WHO Classification of Tumours; IARC Press: Lyon, France, 2017.

6. Ho, P.; Wang, W.C.; Huang, Y.T.; Yang, Y.H. Finding an oral potentially malignant disorder in screening program is related to early diagnosis of oral cavity cancer-Experience from real world evidence. Oral Oncol. 2019, 89, 107-114. [CrossRef] [PubMed]

7. Morikawa, T.; Kosugi, A.; Shibahara, T. The Utility of Optical Instrument "ORALOOK ${ }^{\circledR ”}$ in the Early Detection of High-risk Oral Mucosal Lesions. Anticancer Res. 2019, 39, 2519-2525. [CrossRef] [PubMed]

8. International Union against Cancer. TNM Classification of Malignant Tumours, 8th ed.; WILEY-Blackwell: New York, NY, USA, 2017.

9. Scully, C.; Bagan, J.V.; Hopper, C.; Epstein, J.B. Oral cancer: Current and future diagnostic techniques. Am. J. Dent. 2008, 21, 199-209. [PubMed]

10. Chakraborty, D.; Natarajan, C.; Mukherjee, A. Advances in oral cancer detection. Adv. Clin. Chem. 2019, 91, 181-200.

11. Abdulhameed, H.A.; Omar, K.; Camile, S.; Farah, C.S. The utility of oral brush cytology in the early detection of oral cancer and oral potentially malignant disorders: A systematic review. J. Oral Pathol. Med. 2018, 47, 104-116.

12. Takano, M.; Kakizawa, T.; Takasaki, Y.; Seta, S.; Noma, H.; Yajima, Y.; Nomura, S. Clinical classification to indicate stage of oral precancerous lesions and early cancer with iodine and toluideine bleu staining test. Head Neck Cancer. 2002, 28, 41-46. [CrossRef]

13. Mascitti, M.; Orsini, G.; Tosco, V.; Monterubbianesi, R.; Balercia, A.; Putignano, A.; Procaccini, M.; Santarelli, A. An Overview on Current Non-invasive Diagnostic Devices in Oral Oncology. Front Physiol. 2018, $25,1510$. [CrossRef] [PubMed]

14. Nagi, R.; Reddy-Kantharaj, Y.B.; Rakesh, N.; Janardhan-Reddy, S.; Sahu, S. Efficacy of light based detection systems for early detection of oral cancer and oral potentially malignant disorders: Systematic review. Med. Oral Patol. Oral Circ. Bucal 2016, 21, 447-455. [CrossRef]

15. Morikawa, M.; Kosugi, A.; Bessho, H.; Nomura, T.; Katakura, A.; Shibahara, T. Analysis of tongue squamous cell carcinoma and leukoplakia by optical instrument. J. Jpn. Stomatol. Soc. 2017, 66, 273-282. [CrossRef]

16. Morikawa, M.; Bessho, H.; Kozakai, A.; Kosugi, A.; Shibahara, T. Analysis of oral squamous cell carcinoma and oral lichen planus using the "IllumiScan ${ }^{\circledR "}$ optical instrument. Shikwa Gakuho 2017, 117, 383-392. [CrossRef]

17. Morikawa, T.; Kozakai, A.; Kosugi, A.; Bessho, H.; Shibahara, T. Image processing analysis of oral cancer, oral potentially malignant disorders, and other oral diseases using optical instruments. Int. J. Oral Maxillofac. Surg. 2020, 49, 515-521. [CrossRef] [PubMed]

18. Katakura, A.; Ukiti, K.; Takeda, S.; Kin, M.; Nomura, T.; Yakushiji, T.; Takagi, R.; Shibahara, T. Application of fluorescence visualization Technology to the screening of oral mucosal diseases. J. Jpn. Soc. Laser. Dent. 2012, 23, 142-146. [CrossRef]

19. Guidelines for handling tongue cancer. Japanese Society of Oral Oncology. J. Jpn. Soc. Oral Tumors. 2005, 17, 13-85. [CrossRef]

20. Kusukawa, J.; Suefuji, Y.; Ryu, F.; Noguchi, R.; Iwamoto, O.; Kameyama, T. Dissemination of cancer cells into circulation occurs by incisional biopsy of oral squamous cell carcinoma. J. Oral Pathol. Med. 2000, 29, 303-307. [CrossRef] 
21. Dyavanagoudar, S.; Kale, A.; Bhat, K.; Hallikerimath, S. Reverse transcriptase polymerase chain reaction study to evaluate dissemination of cancer cells into circulation after incision biopsy in oral squamous cell carcinoma. Indian J. Dent Res. 2008, 19, 315-319. [CrossRef]

22. Alsarraf, A.; Kujan, O.; Farah, C.S. Liquid-based oral brush cytology in the diagnosis of oral leukoplakia using a modified Bethesda Cytology system. J. Oral Pathol. Med. 2018, 47, 887-894. [CrossRef]

23. Lena, D.; Kristin, G.; Heinrich, N.; Torsten, W.R. Evaluation of the Accuracy of Liquid-Based Oral Brush Cytology in Screening for Oral Squamous Cell Carcinoma. Cancers 2019, 11, 1813. [CrossRef]

24. Kujan, O.; Pemberton, M.N.; Schwarz, M.; Sloan, P. Evaluation of an innovative oral brush for potential applications using liquid based cytology. J. Oral Sci. 2018, 24, 45-50. [CrossRef]

25. Jajodia, E.; Raphael, V.; Shunyu, N.B.; Ralte, S.; Pala, S.; Jitani, A.K. Brush cytology and AgNOR in the diagnosis of oral squamous cell carcinoma. Acta Cytol. 2017, 61, 62-70. [CrossRef]

26. Navone, R.; Pentenero, M.; Gandolfo, S. Liquid-based cytology in oral cavity squamous cell cancer. Curr. Opin. Otolaryngol. Head Neck Surg. 2011, 19, 77-81. [CrossRef] [PubMed]

27. Epstein, J.B.; Scully, C.; Spinelli, J. Toluidine blue and Lugol's iodine application in the assessment of oral malignant disease and lesions at risk of malignancy. J. Oral Pathol. Med. 1992, 21, 160-163. [CrossRef] [PubMed]

28. Elimairi, I.; Altay, M.A.; Abdoun, O.; Elimairi, A.; Tozoglu, S.; Baur, D.A.; Quereshy, F. Clinical relevance of the utilization of vital Lugol's iodine staining in detection and diagnosis of oral cancer and dysplasia. Clin. Oral Investig. 2017, 21, 589-595. [CrossRef] [PubMed]

29. Petruzzi, M.; Lucchese, A.; Baldoni, E.; Grassi, F.R.; Serpico, R. Use of Lugol's iodine in oral cancer diagnosis: An overview. Oral Oncol. 2010, 46, 811-813. [CrossRef]

30. Epstein, J.B.; Sciubba, J.; Silverman, S., Jr.; Sroussi, H.Y. Utility of toluidine blue in oral premalignant lesions and squamous cell carcinoma: Continuing research and implications for clinical practice. Head Neck. 2007, 29, 948-958. [CrossRef]

31. Chen, Y.W.; Lin, J.S.; Wu, C.H.; Lui, M.T.; Kao, S.Y.; Fong, Y. Application of in vivo stain of methylene blue as a diagnostic aid in the early detection and screening of oral squamous cell carcinoma and precancer lesions. J. Chin. Med. Assoc. 2007, 70, 497-503. [CrossRef]

32. Chhabra, N.; Chhabra, S.; Sapra, N. Diagnostic Modalities for Squamous Cell Carcinoma: An Extensive Review of Literature-Considering Toluidine Blue as a Useful Adjunct. J. Maxillofac. Oral Surg. 2015, 14, 188-200. [CrossRef]

33. Mojsa, I.; Kaczmarzyk, T.; Zaleska, M.; Stypulkowska, J.; Zapala-Pospiech, A.; Sadecki, D. Value of the ViziLite Plus System as a diagnostic aid in the early detection of oral cancer/premalignant epithelial lesions. J. Craniofac. Surg. 2012, 23, e162-e164. [CrossRef]

34. Onofre, M.A.; Sposto, M.R.; Navarro, C.M. Reliability of toluidine blue application in the detection of oral epithelial dysplasia and in situ and invasive squamous cell carcinomas. Oral Surg. Oral Med. Oral Pathol. Oral Radiol. Endod. 2001, 91, 535-540. [CrossRef]

35. Nomura, T.; Shibahara, T. Detection of field alterations using useful tools for oral squamous cell carcinoma. Jpn Dent Sci Rev. 2013, 49, 106-115. [CrossRef]

36. Farah, C.S.; McIntosh, L.; Georgiou, A.; McCullough, M.J. Efficacy of tissue autofluorescence imaging (VELScope) in the visualization of oral mucosal lesions. Head Neck. 2012, 34, 856-862. [CrossRef]

37. Brookner, C.K.; Follen, M.; Boiko, I.; Galvan, J.; Thomsen, S.; Malpica, A.; Suzuki, S.; Lotan, R.; Richards-Kortum, R. Autofluorescence patterns in short-term cultures of normal cervical tissue. Photochem. Photobiol. 2000, 71, 730-736. [CrossRef]

38. Richards-Kortum, R.; Sevick-Muraca, E. Quantitative optical spectroscopy for tissue diagnosis. Annu. Rev. Phys. Chem. 1996, 47, 555-606. [CrossRef]

39. Wagnières, G.A.; Star, W.M.; Wilson, B.C. In vivo fluorescence spectroscopy and imaging for oncological applications. Photochem. Photobiol. 1998, 68, 603-632. [CrossRef] [PubMed]

40. Harada, K.; Ferdous, T.; Harada, T.; Ueyama, Y. Metformin in combination with 5fluorouracil suppresses tumor growth by inhibiting the Warburg effect in human oral squamous cell carcinoma. Int. J. Oncol. 2016, 49, 276-284. [CrossRef] [PubMed]

41. Sekine, R.; Takushiji, T.; Tanaka, Y.; Shibahara, T. A study on the intrapapillary capillary loop detected by narrow band imaging system in early oral squamous cell carcinoma. J. Oral Maxillofac. Surg. Med. Pathol. 2015, 27, 624-630. [CrossRef] 
42. Marioni, G.; Staffieri, A.; Fasanaro, E.; Stramare, R.; Giacomelli, L.; Bernardi, E. The role of angiogenin in pT1-T2 tongue carcinoma neo-angiogenesis and cell proliferation: An exploratory study. J. Oral Pathol. Med. 2013, 42, 606-611. [CrossRef]

43. Scheer, M.; Fuss, J.; Derman, M.A.; Kreppel, M.; Neugebauer, J.; Rothamel, D.; Drebber, U.; Zoeller, J.E. Autofluorescence imaging in recurrent oral squamous cell carcinoma. Oral Maxillofac. Surg. 2016, 20, 27-33. [CrossRef]

44. Scheer, M.; Neugebauer, J.; Derman, A.; Fuss, J.; Drebber, U.; Zoeller, J.E. Autofluorescence imaging of potentially malignant mucosa lesions. Oral Surg. Oral Med. Oral Pathol. Oral Radiol. Endod. 2011, 111, 568-577. [CrossRef]

45. Uchida, H.; Vaidyanathan, J.; Viswanadhan, T.; Vaidyanathan, T.K. Color stability of dental composites as a function of shade. J. Prosthet. Dent. 1998, 79, 372-377. [CrossRef]

46. Rogers, D.F. Procedural Elements for Computer Graphics; McGraw-Hill Inc.: New York, NY, USA, 1988; pp. 383-410.

47. International Commission on Illumination. Available online: http://cie.co.at/ (accessed on 27 April 2020).

48. Kikuta, S.; Iwanaga, J.; Todoroki, K.; Shinozaki, K.; Tanoue, R.; Nakamura, M.; Kusukawa, J. Clinical Application ofthe IllumiScan Fluorescence Visualization Device in Detecting Oral Mucosal Lesions. Cureus. 2018, 6, e3111. [CrossRef]

49. Kozakai, A.; Ono, K.; Nomura, T.; Takano, T.; Shibahara, T. Usefulness of objective evaluations by fluorescence visualization device for differentiating between superficial oral squamous cell carcinoma and oral lichen planus. J. Oral Maxillofac. Surg. Med. Pathol. 2020, 32, 26-32. [CrossRef]

50. Kwon, S.H.; Yoon, J.K.; An, Y.S.; Shin, Y.S.; Kim, C.H.; Lee, D.H.; Jo, K.S.; Lee, S.J. Prognostic significance of the intratumoral heterogeneity of (18) F-FDG uptake in oral cavity cancer. J. Surg. Oncol. 2014, 110, 702-706. [CrossRef]

51. Morikawa, T.; Futoo, E.; Bessho, H.; Yakushiji, T.; Nomura, T.; Onodera, S.; Uchino, Y.; Takano, N.; Shibahara, T. ${ }^{18}$ F-FDG PET/CT parameters as imaging biomarker is useful in oral squamous cell carcinoma patients. J. Jpn. Soc. Oral Tumor J. Jpn. Soc. Oral Oncol. 2017, 29, 23-35. [CrossRef]

52. Kosugi, A.; Kasahara, M.; Yang, L.; Nakamura-takahashi, A.; Shibahara, T.; Mori, T. Method for diagnosing neoplastic lesions by quantitative fluorescence value. Sci. Rep. 2019, 24, 7833. [CrossRef]

53. Güneri, P.; Epstein, J.B.; Ergün, S.; Boyacioğlu, H. Toluidine blue color perception in identification of oral mucosal lesions. Clin. Oral Investig. 2011, 15, 337-345. [CrossRef]

54. Morikawa, T.; Kosugi, A.; Kozakai, A.; Marumo, T.; Bessho, H.; Shibahara, T. Image Analysis using Squamous Cell Carcinoma and Leukoplakia by Optical Instrument. J. Jpn. Soc. Laser Dent. 2019, 29, 131-140.

55. Kar, A.; Wreesmann, V.B.; Shwetha, V.; Thakur, S.; Rao, V.U.S.; Arakeri, G.; Brennan, P.A. Improvement of oral cancer screening quality and reach: The promise of Artificial Intelligence. J. Oral Pathol. Med. 2020, 11. in press. [CrossRef]

56. Sugahara, K.; Futoo, E.; Bessho, H.; Riyo, S.; Ohno, K.; Katakura, A.; Shibahara, T. Fluorescence Visualization for Oral Mucosa Using an Auto-fluorescence Imaging Analysis Software. Jpn. J. Oral Diag. 2017, 30, $168-175$.

57. NIH U.S. National Library of Medicine Clinical Trials. Gov. Direct Visual Fluorescence in Finding Oral Cancer in High-Risk Patients and Patients Undergoing Routine Dental Care. ClinicalTrials.gov Identifier: NCT01816841. Available online: http://cie.co.at/ (accessed on 24 September 2020).

58. NIH U.S. National Library of Medicine Clinical Trials. Gov. Efficacy of Optically-guided Surgery in the Management of Early-staged Oral Cancer-COOLS TRIAL. ClinicalTrials.gov Identifier: NCT01039298. Available online: http://cie.co.at/ (accessed on 24 September 2020).

59. Rasband, W.S. ImageJ, U.S. National Institutes of Health: Bethesda, Maryland, USA, 1997-2012. Available online: http://imagej.nih.gov/ij/ (accessed on 6 April 2020).

60. Schneider, C.A.; Rasband, W.S.; Eliceiri, K.W. NIH Image to ImageJ: 25 years of image analysis. Nat. Methods 2012, 9, 671-675.

(C) 2020 by the authors. Licensee MDPI, Basel, Switzerland. This article is an open access article distributed under the terms and conditions of the Creative Commons Attribution (CC BY) license (http://creativecommons.org/licenses/by/4.0/). 